\title{
Clipping of Lateral Posterior Choroidal Intraventricular Aneurysm Related to Ruptured Temporal Arteriovenous Malformation: Case Report
}

\author{
Jaime López Calle, Rommel Arbulú Zuazo, Ronald Pascual Valverde, \\ Walter Colunga Cáceres, Katya Chávez Barboza, César Fustamante Torres, \\ Yosimar Coasaca Tito, Luis Muñoz Chumbes
}

Neurosurgery Section, Arzobispo Loayza Hospital, Lima, Peru

Email: lopezcallej@gmail.com

Received 3 November 2015; accepted 3 January 2016; published 6 January 2016

Copyright (C) 2016 by authors and Scientific Research Publishing Inc.

This work is licensed under the Creative Commons Attribution International License (CC BY). http://creativecommons.org/licenses/by/4.0/

(c) (i)

\begin{abstract}
Background: Intraventricular cerebral aneurysms are uncommon brain vascular disease in general population. The previous reported cases point with more frequency to Moyamoya disease as the main factor to originate aneurysms in the choroidal arteries. Those related to an AVM are even rarer and only two cases have been previously reported. Aim: To discuss the unusual location of this aneurysm, its relationship to other conditions and the type of treatment. Case Presentation: We present the case of a patient with a non-ruptured intraventricular aneurysm of lateral posterior choroidal artery related to a ruptured right temporal arteriovenous malformation, who was successfully treated by clipping immediately, after resection of the malformation. Conclusion: Intraventricular choroidal artery aneurysm is a rare pathology. Its deep location makes the treatment challenging but mandatory due to the high probability of bleeding, especially those flow-related to an arteriovenous malformation.
\end{abstract}

\section{Keywords}

Posterior Choroidal Artery, Aneurysm, Arteriovenous Malformation, Clipping

\section{Introduction}

Intraventricular cerebral aneurysms are rare neurovascular diseases. To date, only less than 50 cases have been

How to cite this paper: Calle, J.L., et al. (2016) Clipping of Lateral Posterior Choroidal Intraventricular Aneurysm Related to Ruptured Temporal Arteriovenous Malformation: Case Report. Open Journal of Modern Neurosurgery, 6, 9-15. 
reported worldwide, so the few aspects known have made the management of such aneurysms difficult, despite its deep location, small size and peculiar angiographic characteristics. Even rarer are the distal flow-related intraventricular aneurysms in the context of an AVM with only two previous cases reported. Our objective is to review the unusual location of this aneurysm, its relationship to an AVM and other conditions and the type of treatment used in this case.

\section{Case Presentation}

Fifty-nine-year-old female patient went through failed surgery thirty years ago in another hospital. Due to a ruptured right temporal arteriovenous malformation (AVM) she was admitted in our institution. After 14 days receiving initial care in another hospital she was transferred to us. She presented sudden headache, nausea and vomiting and transitory disorder of consciousness. At admission she was awake, oriented and showed no motor deficit, Glasgow Coma Scale (GCS): 15 pts. The Brain Computed Tomography (CT) showed a right temporal intracerebral hemorrhage of 20 cc volume. Cerebral angiography showed the presence of a posterior right temporal AVM Spetzler-Martin grade (SM) II, $40 \times 15 \times 10 \mathrm{~mm}$, with two main feeders: a deep afferent is the lateral posterior choroidal artery, in which way a distal aneurysm $2 \times 7 \mathrm{~mm}$ was observed (Figure 1 and Figure 2) and the other is the anterior temporal artery (Figure 3 and Figure 4); plus small dural feeders of the superficial temporal artery with one superficial drainage vein to the sigmoid sinus.

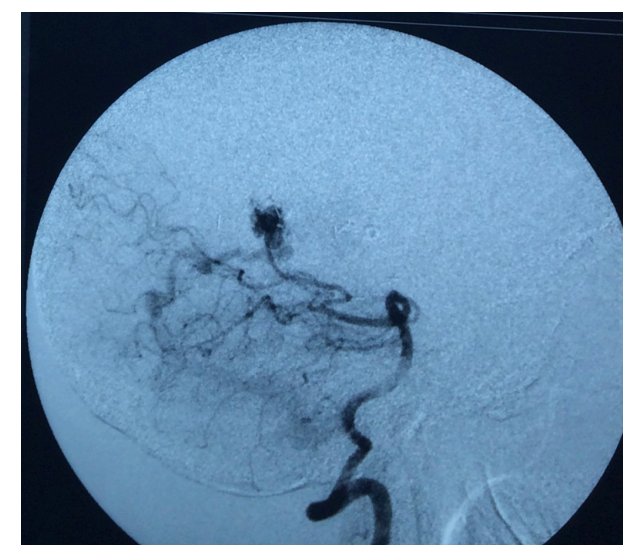

Figure 1. Preoperative angiography demonstrating a posterior temporal AVM SM II has two contiguous nidus fed by the anterior temporal artery and lateral posterior choroidal artery.

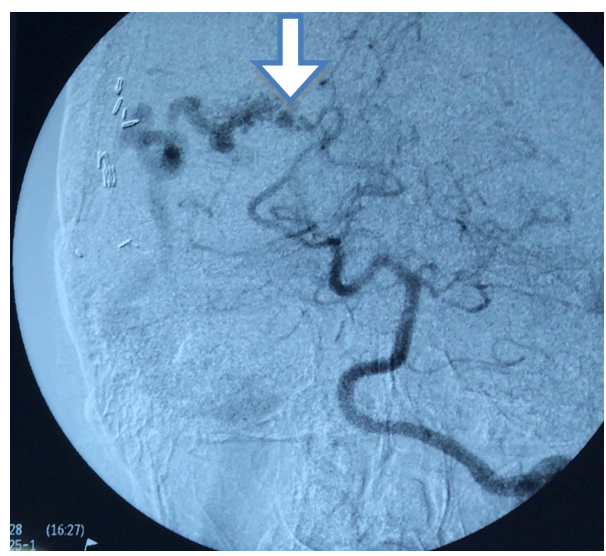

Figure 2. Preoperative angiography demonstrating a posterior temporal AVM SM II has two contiguous nidus fed by the anterior temporal artery and lateral posterior choroidal artery. Note: the presence of the aneurysm in the distal part of the lateral posterior choroidal artery just before the nidus. 


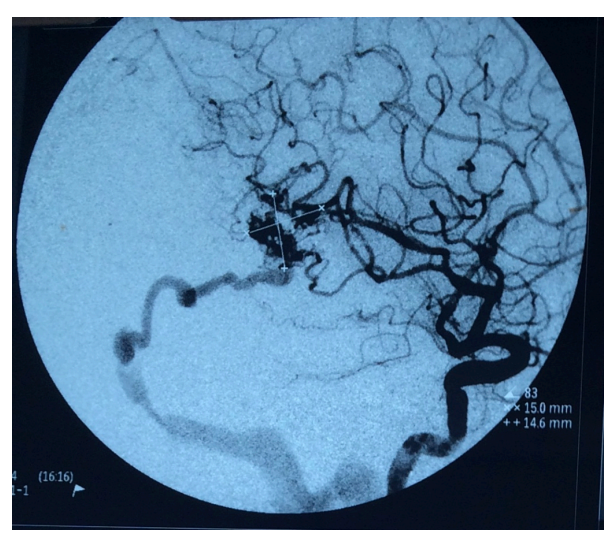

Figure 3. Preoperative angiography demonstrating a posterior temporal AVM SM II has two contiguous nidus fed by the anterior temporal artery and lateral posterior choroidal artery.

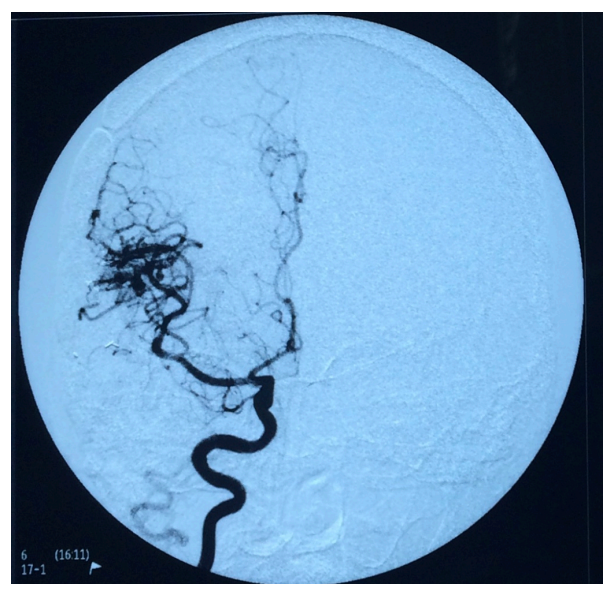

Figure 4. Preoperative angiography demonstrating a posterior temporal AVM SM II has two contiguous nidus fed by the anterior temporal artery and lateral posterior choroidalartery.

After reopening the right temporoparietal craniotomy, the dura was opened under microscope and an extensive scar tissue and some older clips were observed over the cortex. First we coagulated the dural feeders under meticulous micro dissection; after we evacuated the clot, the AVM was resected. Finally, we found a lateral posterior choroidal intraventricular aneurysm just adjacent to the ventricular wall (Figure 5) that was clipped (Mizuho Inc. Sugita clip). An intraoperative angiography was done without residual AVM and appropriate clipping of the aneurysm (Figures 6-9). Postoperative neurologic outcome was favorable, with 24 hs postoperative brain CT showed no presence of infarcts and clip in the right occipital horn. Also the patient presented sepsis due to cellulitis, which improved providing antibiotics. She was discharged two weeks later. At the late follow up (6 months) the patient is in good functional outcome according to the Rankin modified scale (mRS 2).

\section{Discussion}

Association between cerebral arteriovenous malformation (AVM) and intracranial aneurysm is known and presentation percentages widely varies between $2.7 \%$ to $58 \%$; however, the most frequent values reported range between $10 \%$ to $20 \%$. [1] [3]. It is also well established that presence of these aneurysms increases the risk of bleeding [2] [3].

Redekop et al. classified the aneurysms related to an AVM as intranidal, related to flow (proximal or distal) and unrelated [4], as well as he found that intranidal aneurysms increases the likelihood of bleeding up to 9.8\% and flow-related aneurysms reaches 5.3\% per year, compared to $2 \%-4 \%$ attributed to an AVM. Nevertheless, 


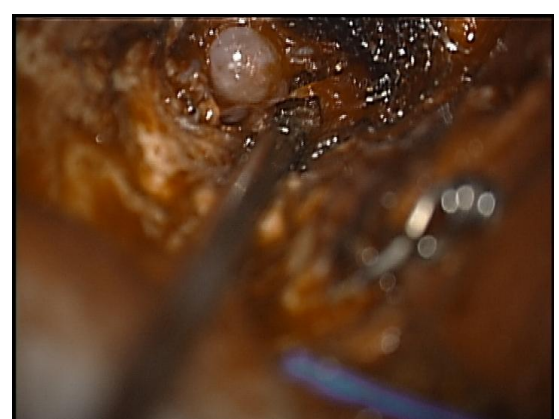

Figure 5. Intraoperative image of the lateral posterior choroidal artery aneurysm in the deep just adjacent to the ventricular wall.

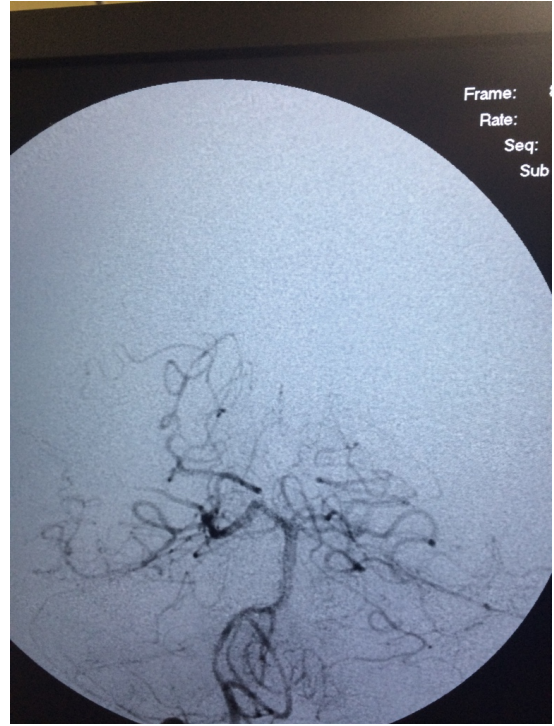

Figure 6. Intraoperative angiography demonstrating no residual AVM and adequate closure of the aneurysm.

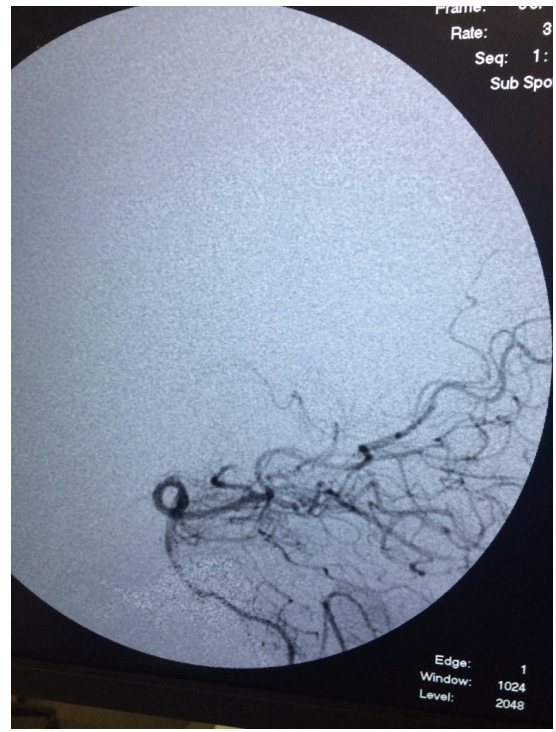

Figure 7. Intraoperative angiography demonstrating no residual AVM and adequate closure of the aneurysm. 


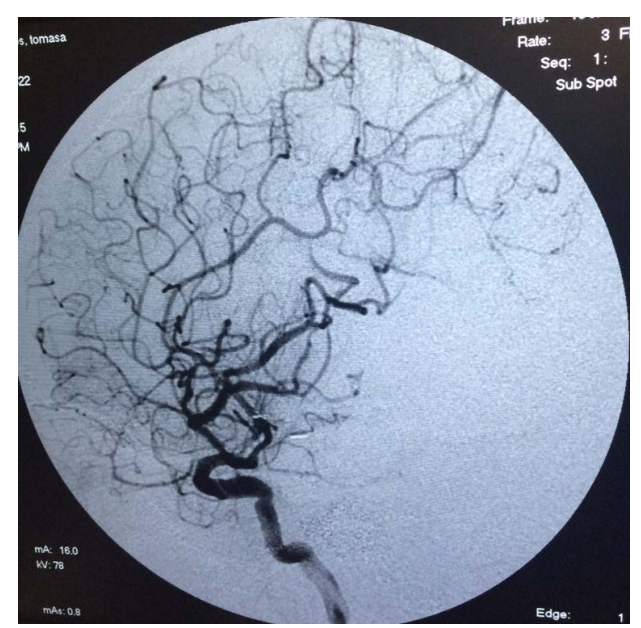

Figure 8. Intraoperative angiography demonstrating no residual AVM and adequate closure of the aneurysm.

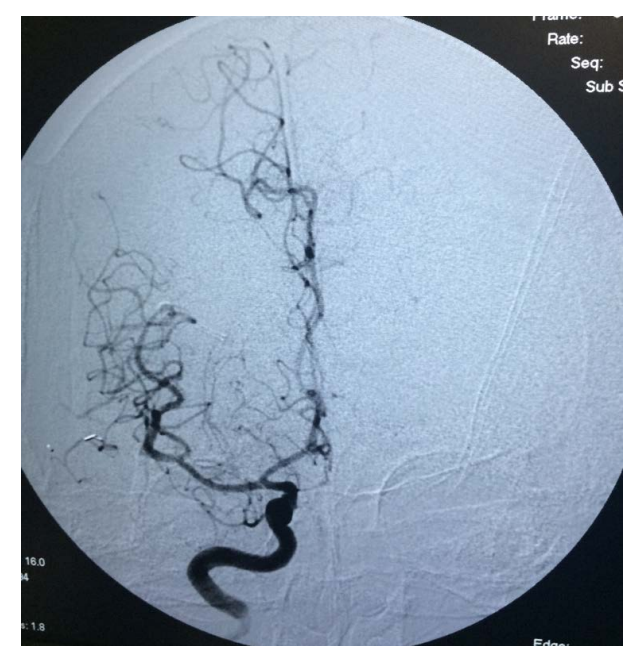

Figure 9. Intraoperative angiography demonstrating no residual AVM and adequate closure of the aneurysm.

during the monitoring period could not prove in any patient that the source of the bleeding was aneurysmatic. Instead, Batger et al. [5] found that 78\% of patients studied, had bled from a distal flow-related aneurysm. Similarly, Kim et al. [6] found that $16 \%$ of 138 patients with hemorrhagic presentation had extranidal flow-related aneurysm bleeding, being more related distal aneurysms to intracerebral bleeding compared to proximal. Giuseppe D'Aliberti et al. [7], in a recent report found similar finding, added to the postnidal venous aneurysms the same potential bleeding.

Intraventricular aneurysms are rare pathologies and usually the few reported cases are associated with Moyamoya disease [8]-[11], due to the great hemodinamic stress that affects the collateral arteries and pathological vessel architecture, among the ones can find choroidal arteries.

Reports of these aneurysms point out more frequently distal anterior choroidal artery aneurysms, some related to Moyamoya disease [8]-[11], to an arteriovenous malformation [12] and others in isolation without any other pathology added or so called idiopathic [13]-[15].

The distal and intraventricular posterior choroidal artery aneurysms are even rarer than such mentioned. So much so that to date 22 cases of posterolateral choroidal artery aneurysms have been published worldwide: 16 of them associated with Moyamoya, 4 idiopathic and only 2 associated with arteriovenous malformation [8]. To our knowledge our case would be the third associated to an AVM. The oldest case reported on distal intraventricular 
aneurysm of the posterior choroidal artery is located in PubMed and published by UngersbiickPerneczky [16]; but unlike the present case, the location was in the medial posterior choroidal, not on the rear side.

Although some reports considered conservative management as an alternative to be branded as "pseudoaneurysm" [17] or even they could be disappear after treatment of the AVM [18] now is known to produce high probability of bleeding and/or rebleeding and the consequent likelihood of death or disability, so its active microsurgical or endovascular management is recommended.

Microsurgical management includes: clipping, resection or trapping; with the risk of parenchymal damage due to transcortical or transcallosal approach to treat a deep lesion and in the case of Moyamoya disease with many important collateral arteries near the aneurysm.

The endovascular option is attractive because it avoids manipulation of the parenchyma, but sometimes it is difficult to catheterize arteries because of their size, many loops or fragile aneurysms walls more susceptible to rupture with coils.

In this case the microsurgical decision was taken because it is a flow-related aneurysm associated to a ruptured arteriovenous malformation which required surgical treatment and was located close to the nidus in the deep part, so that at the end of surgery after resection of the vascular malformation we proceeded to clip the aneurysm on a direct position towards our approach with good clinical and radiological results.

\section{Conclusion}

Intraventricular lateral posterior choroidal artery aneurysm is a rare pathology. Its deep location makes the treatment challenging but mandatory due to the high probability of bleeding, especially those flow-related to an arteriovenous malformation.

\section{References}

[1] Flores, B.C., Klinger, D.R., Rickert, K.L., Barnett, S.L., Welch, B.G., White, J.A., Batjer, H.H. and Samson, D.S. (2014) Management of Intracranial Aneurysms Associated with Arteriovenous Malformations. Neurosurgical Focus, 37, E11. http://dx.doi.org/10.3171/2014.6.focus14165

[2] Almefty, K. and Spetzler, R.F. (2011) Arteriovenous Malformations and Associated Aneurysms. World Neurosurgery, 76, 396-397. http://dx.doi.org/10.1016/j.wneu.2011.06.051

[3] Lv, X., Wu, Z., Li, Y., Jiang, C., Yang, X. and Zhang, J. (2011) Cerebral Arteriovenous Malformations Associated with Flowrelated and Circle of Willis Aneurysms. World Neurosurgery, 76, 455-458. http://dx.doi.org/10.1016/j.wneu.2011.04.015

[4] Redekop, G., TerBrugge, K., Montanera, W. and Willinsky, R. (1998) Arterial Aneurysms Associated with Cerebral Arteriovenous Malformations: Classification, Incidence, and Risk of Hemorrhage. Journal of Neurosurgery, 89, 539546. http://dx.doi.org/10.3171/jns.1998.89.4.0539

[5] Batjer, H., Suss, R.A. and Samson, D. (1986) Intracranial Arteriovenous Malformations Associated with Aneurysms. Neurosurgery, 18, 29-35. http://dx.doi.org/10.1227/00006123-198601000-00006

[6] Kim, E.J., Halim, A.X., Dowd, C.F., Lawton, M.T., Singh, V., Bennett, J. and Young, W. (2004) The Relationship of Coexisting Extranidal Aneurysms to Intracranial Hemorrhage in Patients Harboring Brain Arteriovenous Malformations. Neurosurgery, 54, 1349-1358. http://dx.doi.org/10.1227/01.neu.0000124483.73001.12

[7] D'Aliberti, G., Talamonti, G., Cenzato, M., La Camera, A., Debernardi, A., Valvassori, L., Mariangela, P. and Nichelatti, M. (2015) Arterial and Venous Aneurysms Associated with Arteriovenous Malformations. World Neurosurgery, 83, 188-196. http://dx.doi.org/10.1016/j.wneu.2014.05.037

[8] Zhu, Y., Zhao, W.H. and Xu, W.M. (2013) Intraventricular Aneurysms: Case Reports and Review of the Literature. Clinical Neurology and Neurosurgery, 115, 57-64. http://dx.doi.org/10.1016/j.clineuro.2012.04.014

[9] He, K., Zhu, W., Chen, L. and Mao, Y. (2013) Management of Distal Choroidal Artery Aneurysms in Patients with Moyamoya Disease: Report of Three Cases and Review of the Literature. World Journal of Surgical Oncology, $11,187$. http://dx.doi.org/10.1186/1477-7819-11-187

[10] Yang, S., Yu, J.-L., Wang, H.-L., Wang, B. and Luo, Q. (2010) Endovascular Embolization of Distal Anterior Choroidal Artery Aneurysms Associated with Moyamoya Disease. A report of Two Cases and a Literature Review. Interventional Neuroradiology, 16, 433-441.

[11] Miyake, H., Ohta, T., Kajimoto, Y., Ogawa, R. and Deguchi, J. (2000) Intraventricular Aneurysms. Three Case Reports. Neurologia Medico-Chirurgica (Tokyo), 40, 55-60. http://dx.doi.org/10.2176/nmc.40.55 
[12] Yanaka, K., Tsuboi, K., Fujita, K., Aoki, K., Takeuchi, S., Anno, I. and Nose, T. (2000) Distal Anterior Choroidal Artery Aneurysm Associated with an Arteriovenous Malformation. Intraoperative Localization and Treatment. Surgical Neurology, 53, 546 -51. http://dx.doi.org/10.1016/S0090-3019(00)00235-4

[13] Pavesi, G., Amistá, P., Munari, M. and Gardiman, M.P. (2008) Intraventricular Hemorrhage Caused by Peripheral Anterior Choroidal Artery Aneurysm Rupture. A Case Report. The Neuroradiology Journal, 21, 717-720. http://dx.doi.org/10.1177/197140090802100518

[14] Inci, S., Arat, A. and Ozgen, T. (2007) Distal Anterior Choroidal Artery Aneurysms. Surgical Neurology, 67, 46-52. http://dx.doi.org/10.1016/j.surneu.2006.05.053

[15] Yurt, A., Turan, Y., Ucar, K., Camlar, M. and Oran, I. (2009) Ruptured Distal Anterior Choroidal Artery Aneurysm. Journal of Clinical Neuroscience, 16, 132-134. http://dx.doi.org/10.1016/j.jocn.2008.02.018

[16] Ungersbiick, K. and Perneczky, A. (1986) Intraventricular Aneurysm of the Medial Posterior Choroid Artery Clipped via the Contralateral Transcallosal Approach. Acta Neurochirurgica (Wien), 82, 24-27. http://dx.doi.org/10.1007/BF01456315

[17] Hamada, J., Hashimoto, N. and Tsukahara, T. (1994) Moyamoya Disease with Repeated Intraventricular Hemorrhage due to Aneurysm Rupture. Report of Two Cases. Journal of Neurosurgery, 80, 328-331. http://dx.doi.org/10.3171/jns.1994.80.2.0328

[18] He, L., Gao, J.W., Thomas, A.J., Fusco, M.R. and Ogilvy, C.S. (2015) Disappearance of a Ruptured Distal FlowRelated Aneurysm after Arteriovenous Malformation Nidal Embolization. World Neurosurgery, 84, 1496 E1-E6.

\section{Abbreviations}

AVM: arteriovenous malformation; GCS: Glasgow Coma Scale; CT: Computed Tomography; SM: SpetzlerMartin; modified Rankin Scale: mRS. 